\title{
FITOSSOCIOLOGIA DA VEGETAÇÃO ARBÓREA DA RESERVA FLORESTAL DO SACAVÉM, SÃO LUÍS, MARANHÃO (BRASIL).
}

\author{
Francisca Helena MUNIZ'; Oswaldo CESAR²; Reinaldo MONTEIRO²
}

\begin{abstract}
RESUMO - Foi amostrada fitossociologicamente uma área de 0,5 ha de mata na Reserva Florestal do Sacavém, distando cerca de $7 \mathrm{Km}$ do centro da cidade de São Luís (2"32'S; 44"17'W), em manchas remanescentes de floresta tropical úmida, denominada localmente de "Pré-Amazônia". A área foi dividida em 50 parcelas de $10 \times 10 \mathrm{~m}\left(100 \mathrm{~m}^{2}\right)$, nas quais foram amostrados 410 indivíduos com PAP a partir de 15 $\mathrm{cm}$, pertencentes a 34 famîias, 66 gêneros e 110 espécies, além de 5 indivíduos mortos não considerados na análise fitossociológica. O DAP variou de $4,8 \mathrm{~cm}$ a $86,7 \mathrm{~cm}$, ficando a média em $16,28 \mathrm{~cm}$. As alturas mínima, média e máxima foram, respectivamente, $2,0 \mathrm{~m}, 11,16 \mathrm{~m}$ e $25,0 \mathrm{~m}$. A área basal total foi de 14,207 $\mathrm{m}^{2}$, equivalente a $28,4140 \mathrm{~m}^{2} / \mathrm{ha}$, com média de $0,07 \mathrm{~m}^{2} /$ indivíduo, e a densidade absoluta foi de 820 indivíduos/ha. As cinco familias mais importantes em IVI foram Leguminosae, Chrysobalanaceae, Meliaceae, Myrtaceae e Arecaceae, perfazendo 49,24\% do total. As primeiras cinco espécies em IVI foram Licania cf. incana Aubl., Guarea guidonia (L.) Sleumer, Copaifera langsdorlfii Desf., Dipteryx lacunifera Ducke e uma morto-espécie (Desconhecida 1). Cerca de $60 \%$ das espécies consideradas apresentaram apenas 1 ou 2 indivíduos. $\mathrm{O}$ índice de diversidade de Shannon e Weaver $\left(\mathrm{H}^{\prime}\right)$ foi de 4,189. Foram feitas comparações com diversos trabalhos realizados em florestas amazônicas, confimando que a área em esludo é comparável àquelas em riqueza, densidade e dominância, embora a área tenha sido subdimensionada.
\end{abstract}

Palavras-chave: Fitossociologia, Floresta "Pré-Amazônica", Reserva Florestal do Sacavém (São Luís-MA). Phytosociology of a woody vegetation in the Sacavem Forest Reserve - São Luis, State of Maranhão(Brazil).

ABSTRACT - A phytosociological study was carried out in 0.5 ha of remaining patches of 'Pre-Amazonian' humid tropical forest within the Sacavém Forest Reserve, located in the municipality of São Luís, state of Maranhão, Brazil $\left(2^{\circ} 32^{\prime} \mathrm{S} ; 44^{\circ} 17^{\prime} \mathrm{W}\right)$. The total area comprised of fifty $10 \times 10 \mathrm{~m}$ quadrats in which 410 trees with a minimum perimeter at breast height $(\mathrm{PBH})$ of $15 \mathrm{~cm}$ were sampled, including 5 dead individuals. There were found 110 species of 66 genera in 34 families. The DBH varied between 4.8 $\mathrm{cm}$ and $86.7 \mathrm{~cm}$, with an average of $16.28 \mathrm{~cm}$. The minimum, mean and maximum heights were $2.0 \mathrm{~m}$, $11.16 \mathrm{~m}$ and $25.0 \mathrm{~m}$, respectively. The total basal area was $14.207 \mathrm{~m}^{2}$ or $28.4140 \mathrm{~m} / \mathrm{ha}$ with an average of $0,07 \mathrm{~m}^{2} / \mathrm{tree}$, the absolute density was 820 trees/ha. The five most importante families in IVI were Leguminosae, Chrysobalanaceae, Meliaceae, Myrtaceae and Arecaceae, making up 49.24\% of the total. The five most important species in IVI were Licania ct. incana Aubl., Guarea guidonia (L.) Sleumer, Copaifera langsdorffii Dest., Dipteryx lacunifera Ducke and one morpho-species (Unknown 1). As much as $60 \%$ of the species had only one or two individuals. The Shannon \& Weaver diversity index $\left(H^{\prime}\right)$ was 4.189. Although the area studied is comparatively small, several comparisons made with surveys for other Amazonian forest locations led to the conclusion that the Sacavém Reserve has similar richness, density and dominance.

Key-words: Phytosociology, 'Pre-Amazonian' forest, Sacavém Forest Reserve (São Luís, Maranhão).

\section{INTRODUÇÃO}

O Estado do Maranhão, e particularmente o município de São Luís, apresenta uma lacuna quase completa, no que diz respeito ao conhecimento de sua flora e da estrutura de suas formações vegetais. Esta área de extrema importância, uma vez que aí se encontra o extremo oriental da floresta amazônica, havendo

Depto. de Biologia, Univ. Estadual do Maranhão, Cidade Universitária Paulo VI, Tirirical, CEP 65000-000, São Luís, MA; 2. Depto. Botânica, Inst. Biociências, UNESP, AV. 24A, n. 1515, Caixa Postal 199, CEP 13506-900, Rio Claro,SP 
ligação com as Províncias Central e Atlântica, se constitui, segundo OREN (1988), numa das áreas menos conhecidas do País em sua biogeografia.

O entendimento do papel das diferentes espécies na comunidade, assim como a sociabilidade específica são de grande importância para o conhecimento mais detalhado da dinâmica dessas formações vegetais. $\mathrm{O}$ presente trabalho, realizado em uma área coberta originalmente por floresta tropical úmida, atualmente restrita a pequenas manchas separadas por floresta secundária, foi conduzido com o propósito de fornecer dados sobre a estrutura fitossociológica da Reserva Florestal do Sacavém, visando contribuir para a conservação e preservação da cobertura vegetal dessas áreas, em detrimento de sua ocupação e total destruição.

\section{MATERIAL E MÉTODOS}

A caracterização da área de estudo e as informações de clima e solo são apresentadas por MUNIZ et cl. (1994).

O estudo fitossociológico foi realizado utilizando-se o método de parcelas (BRAUN-BLANQUET, 1979). Foram estabelecidas em 4 áreas não contíguas no interior da mata de terra firme 50 parcelas de $10 \times 10 \mathrm{~m}$, totalizando $5.000 \mathrm{~m}^{2}$. Em cada uma das parcelas foram considerados todos os indivíduos lenhosos com perímetro à altura do peito (PAP) igual ou maior que $15 \mathrm{~cm}$, que foram etiquetados, e tiveram sua altura estimada tomandose como referência uma vara de coleta, marcada de metro em metro, com 12 $\mathrm{m}$ de comprimento.

As coletas realizadas mensalmente (de janeiro de 1992 a junho de 1993), foram de material botânico reprodutivo e/ou vegetativo que, após herborização, foi utilizado para identificação. As árvores que não floresceram durante o período de coleta, tiveram sua identificação feita por comparação, através de amostras dos ramos inférteis.

As exsicatas estão depositadas parte no Herbário HRCB do Instituto de Biociências de Río Claro (UNESP) e, parte no Herbário da Universidade Estadual do Maranhão.

O sistema de classificação adotado para as famílias foi o de CRONQUIST (1981), com exceção da família Leguminosae, que optou-se por ENGLER (1964).

Estimou-se os parâmetros fitossociológicos de densidade total (DT), densidade absoluta (DAs) e relativa (DRs), frequência absoluta (FAs) e relativa (FRs), dominância absoluta (DoAs) e relativa (DoRs), área basal total (ABT) e individual (ABI), e os índices de valor de importância (IVI), e de valor de cobertura (IVC) descritos em MUELLER-DOMBOIS \& ELLENBERG (1974), Os resultados foram trabalhados segundo o Programa FITOPAC, de autoria do Dr. George John Sheppherd, do Departamento de Botânica do Instituto de Biologia da Universidade Estadual de Campinas (UNICAMP ), sendo utilizado para os cálculos um microcomputador 386 DX.

A curva espécie/área foi feita somando-se o número de espécies no- 
vas em cada parcela, sorteadas aleatoriamente, com a finalidade de se estimar a suficiência da amostragem.

\section{RESULTADOS}

No presente estudo foram amostrados 410 indivíduos vivos, pertencentes a 88 espécies, 66 gêneros e 34 famílias. Trinta e seis indivíduos pertencentes a 12 famílias foram identificados apenas a nível desse táxon, enquanto que em 20 árvores amostradas não foi possível nenhum tipo de identificação, sendo esses indivíduos separados em 6 morfoespécies. Os 5 indivíduos mortos em pé amostrados não foram considerados na análise fitossociológica. A densidade total (DT) foi de 820 indivíduos/ha, a área basal total de $14,207 \mathrm{~m}^{2}$, correspondendo a $28,4140 \mathrm{~m}^{2} /$ ha e a frequiência total igual a 610,0 .

$\mathrm{Na}$ Tabela I, estão listadas as espécies amostradas por ordem decrescente do índice do valor de importância (IVI) e seus respectivos parâmetros fitossociológicos. As autoridades das espécies estão citadas em MUNIZ et al. (1994).

\section{Densidade Absoluta (DAs)}

As maiores densidades de indivíduos por hectare pertencem a Guarea guidonia (48), Licania cf. incana (46) e Copaifera langsdorffii (44) que contribuem com $16,83 \%$ da densidade total de árvores da fitocenose. Em seguida, com densidades menores, aparecem Protium heptaphyllum e uma espécie cuja família é desconhecida (Desconhecida 1), ambas com 30, Dipteryx lacunifera
(28), Copaifera officinalis e Cecropia palmata, ambas com 26, Maximiliana maripa (24) e Coccoloba pichuna (20). Essas 10 espécies, somadas com as contribuições das três primeiras, atingem $39,27 \%$ da densidade total. Das 110 espécies consideradas, 46 $(41,82 \%)$ apresentaram densidade absoluta de 1 árvore cada, totalizando 92 indivíduos/ha $(11,22 \%)$.

\section{Frequiência Absoluta (FAs)}

A maioria das espécies amostradas (90 espécies, equivalente a $81,82 \%$ ) ocorreu com freqüência absoluta abaixo de $10,0 \%$. Com freqüência absoluta entre 10,0 e 20,0\% foram registradas 16 espécies: Licania cf. incana, Copaifera langsdorffii, Dipteryx lacunifera, Maximiliana maripa, Cecropia palmata, Inga alba, Licania kunthiana, Coccoloba pichuna, Myrcia amazonica, Hymenaea parvifolia, Miconia cf. serialis, uma espécie de Lecythidaceae, Ocotea cujumari, Annona montana, Aspidosperma auriculatum e Myrcia neesiana. Apenas 4 espécies tiveram frequiência absoluta maior que 20,0\%: Guarea guidonia (26,0\%), Protium heptaphyllum (24,0\%), Copaifera officinalis $(22,0 \%)$ e uma morfo-espécie (Desconhecida 1) (22,0\%).

\section{Freqüência (FR), Densidade (DR) e Dominância Relativas (DoR)}

A Figura 1 mostra a distribuição dos valores de frequência (IA), densidade (1B) e dominância (1C) relativas das espécies amostradas por família, perfazendo mais de $80,0 \%$ do total desses parâmetros. Verifica-se que a família Leguminosae dá a maior 
Tabela 1. Espécies amostradas na Reserva Florestal do Sacavém, São Luís - MA, e seus parâmetros fitossociológicos. $\mathrm{n}=$ número de indivíduos; DA = densidade absoluta; $\mathrm{FA}_{\mathrm{s}}=$ frequência absoluta; $\mathrm{AB}_{\mathrm{s}}=$ área basal; $\mathrm{DoA}=$ dominância absoluta; $\mathrm{DR}_{\mathrm{s}}=$ densidade relativa; $\mathrm{Fr}_{\mathrm{s}}=$ frequência relativa; $\mathrm{DoR}=$ dominância relativa; IVI = índice de valor de importância; IVC'= índice de valor de cobertura. As espécies estão ordenadas em ordem decrescente de IVI.

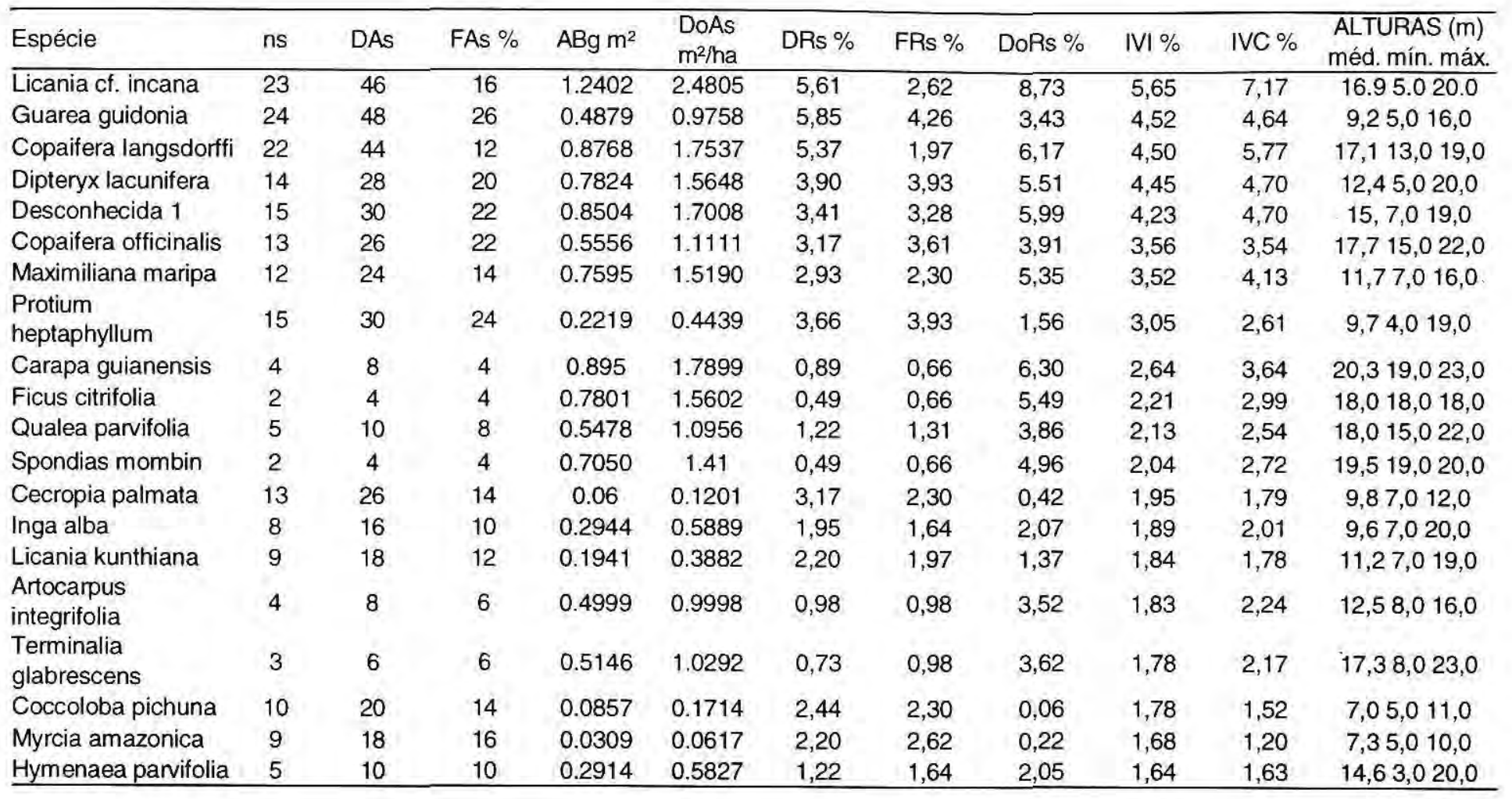


cont. Tabela 1.

\begin{tabular}{|c|c|c|c|c|c|c|c|c|c|c|c|}
\hline Espécie & ns & DAs & FAs $\%$ & $\mathrm{ABg} \mathrm{\textrm {m } ^ { 2 }}$ & $\begin{array}{l}\text { DoAs } \\
\mathrm{m}^{2} / \mathrm{ha}\end{array}$ & DRs $\%$ & FRs $\%$ & DoRs \% & IVI \% & IVC \% & $\begin{array}{l}\text { ALTURAS (m) } \\
\text { méd. mín. máx. }\end{array}$ \\
\hline Pterocarpus rohrii & 2 & 4 & 4 & 0.4540 & 0.9080 & 0,49 & 0,66 & 3,20 & 1,45 & 1,84 & $22,520,025,0$ \\
\hline Miconia cf. serialis & 8 & 16 & 12 & 0.0282 & 0.0564 & 1,95 & 1,97 & 0,20 & 1,37 & 1,07 & $7,85,011,0$ \\
\hline Lecythidaceae & 8 & 16 & 12 & 0.0242 & 0.0484 & 1,95 & 1,97 & 0,17 & 1,36 & 1,06 & $7,55,010,0$ \\
\hline Ocotea cujumari & 5 & 10 & 10 & 0.1746 & 0.3491 & 1,22 & 1,64 & 1,23 & 1,36 & 1,22 & $14,411,018,0$ \\
\hline Annona montana & 7 & 14 & 10 & 0.0642 & 0.1283 & 1,71 & 1,64 & 0,45 & 1,27 & 1,08 & $7,94,0 \quad 13,0$ \\
\hline $\begin{array}{l}\text { Himatanthus } \\
\text { articulatus }\end{array}$ & 5 & 10 & 8 & 0.1492 & 0.2983 & 1,22 & 1,31 & 1,05 & 1,19 & 1,13 & $13,88,016,0$ \\
\hline $\begin{array}{l}\text { Aspidosperma } \\
\text { auriculatum }\end{array}$ & 5 & 10 & 10 & 0.1022 & 0.2044 & 1,22 & 1,64 & 0,72 & 1,19 & 0,97 & $11,62,022,0$ \\
\hline Senna sylvestris & 6 & 12 & 8 & 0.0448 & 0.0896 & 1,46 & 1,31 & 0,32 & 1,03 & 0,89 & $8,77,010,0$ \\
\hline Mouriri cearensis & 5 & 10 & 8 & 0,0639 & 0.1278 & 1,22 & 1,31 & 0,45 & 0,99 & 0,83 & $10,69,012,0$ \\
\hline Myrcia cuprea & 6 & 12 & 8 & 0.0257 & 0.0515 & 1,46 & 1,31 & 0,18 & 0,98 & 0,82 & $7,35,010,0$ \\
\hline Myrcia neesiana & 5 & 10 & 10 & 0.0115 & 0.0231 & 1,22 & 1,64 & 0,08 & 0,98 & 0,65 & $5,85,07,0$ \\
\hline Myrtaceae & 5 & 10 & 8 & 0.0540 & 0.1081 & 1,22 & 1,31 & 0,38 & 0,97 & 0,80 & $11,88,017,0$ \\
\hline $\begin{array}{l}\text { Actinostemon } \\
\text { concepciones }\end{array}$ & 6 & 12 & 8 & 0.0162 & 0.0325 & 1,46 & 1,31 & 0,11 & 0,96 & 0,79 & $6,35,08,0$ \\
\hline Orbygnia phalerata & 3 & 6 & 6 & 0.1622 & 0.3244 & 0,73 & 0,98 & 1,14 & 0,95 & 0,93 & $20,020,020,0$ \\
\hline $\begin{array}{l}\text { Campomanesia } \\
\text { aromatica }\end{array}$ & 5 & 10 & 8 & 0.0455 & 0.0910 & 1,22 & 1,31 & 0,32 & 0,95 & 0,77 & $9,47,012,0$ \\
\hline $\begin{array}{l}\text { Leguminosae } \\
\text { Caesalpinioideae }\end{array}$ & 1 & 2 & 2 & 0.3187 & 0.6374 & 0,24 & 0,33 & 2,24 & 0,94 & 1,24 & $20,020,020,0$ \\
\hline $\begin{array}{l}\text { Enterolobium } \\
\text { schomburgkii }\end{array}$ & 3 & 6 & 4 & 0.1833 & 0.3665 & 0,73 & 0,66 & 1,29 & 0,89 & 1,01 & $13,39,016,0$ \\
\hline Meliaceae & 4 & 8 & 6 & 0.0970 & 0.1939 & 0,98 & 0,98 & 0,68 & 0,88 & 0,83 & $11,57,019,0$ \\
\hline Byrsonima laxiflora & 2 & 4 & 4 & 0.2086 & 0.4172 & 0,49 & 0,66 & 1,47 & 0,87 & 0,98 & $16,513,020,0$ \\
\hline $\begin{array}{l}\text { Pogonophora } \\
\text { schomburgkiana }\end{array}$ & 3 & 6 & 6 & 0.0793 & 0.1585 & 0,73 & 0,98 & 0,56 & 0,76 & 0,64 & $10,77,013,0$ \\
\hline Talisia sp. & 2 & 4 & 4 & 0.1113 & 0.2227 & 0,49 & 0,66 & 0,78 & 0,64 & 0,63 & $14,012,016,0$ \\
\hline Annona sp. & 1 & 2 & 2 & 0.1901 & 0.3802 & 0,24 & 0,33 & 1,34 & 0,64 & 0,79 & $19,019,019,0$ \\
\hline
\end{tabular}




\begin{tabular}{|c|c|c|c|c|c|c|c|c|c|c|c|}
\hline 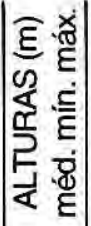 & 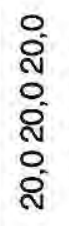 & $\begin{array}{llll}0 & 0 & 0 & 0 \\
\infty & 0 & N & 0 \\
0 & 0 & 0 & 0 \\
\dot{N} & N & N & 0 \\
m & N & N & N \\
0 & \infty & \infty & N\end{array}$ & $\begin{array}{l}0 \\
0 \\
0 \\
0 \\
10 \\
n \\
n\end{array}$ & $\begin{array}{l}0 \\
\infty \\
0 \\
N \\
N \\
N\end{array}$ & $\begin{array}{l}0 \\
0 \\
0 \\
10 \\
10\end{array}$ & 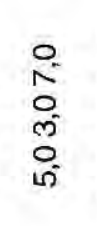 & 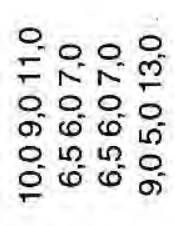 & $\begin{array}{l}0 \\
\text { o } \\
0 \\
\text { 15 } \\
0 \\
\text { N }\end{array}$ & $\begin{array}{llll}0 & 0 & 0 & 0 \\
0 & 0 & 0 & 0 \\
0 & 0 & 0 & 0 \\
0 & 0 & 0 & 0 \\
0 & 0 & 0 & 0 \\
0 & 0 & 0 \\
0 & 0 & 0 & N\end{array}$ & $\begin{array}{ll}0 & 0 \\
\infty & 0 \\
0 & 0 \\
\text { in } & 0 \\
\text { in } & 0 \\
0 & 0\end{array}$ & $\begin{array}{l}0 \\
0 \\
0 \\
0 \\
0 \\
0\end{array}$ \\
\hline \begin{tabular}{l}
0 \\
\hdashline
\end{tabular} & ֻே & 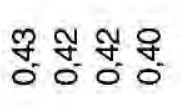 & 웅 & $\stackrel{n}{0}$ & ¿্ষ & लె & 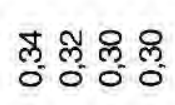 & ల్లి & 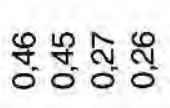 & $\begin{array}{l}\stackrel{0}{\mathrm{~N}} \\
0 \\
0\end{array}$ & ़ֻ \\
\hline$\stackrel{00}{\geq}$ & $\dddot{\wp}$ & $\begin{array}{llll}\overline{6} & \overline{6} & 0 \\
0 & 0 & 0 & 0\end{array}$ & $\begin{array}{l}8 \\
0\end{array}$ & กิ & @্ & 京 & 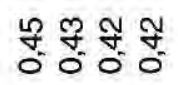 & Yั & 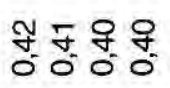 & 웡 웅 & 8 \\
\hline $\begin{array}{l}0 \\
\text { ñ } \\
\frac{1}{8}\end{array}$ & 8 & $\frac{m}{0} \frac{N}{0}=\frac{8}{0}$ & 今̊ & $\frac{\infty}{0}$ & $\begin{array}{l}8 \\
0 \\
0\end{array}$ & L & $\frac{\mathrm{N}}{0} \frac{1}{0} \frac{N}{0}=$ & $\overline{0}$ & 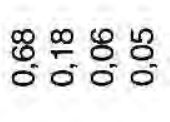 & & $\begin{array}{l}8 \\
0\end{array}$ \\
\hline $\begin{array}{l}\frac{0}{02} \\
\frac{n}{4}\end{array}$ & लू & 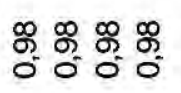 & ô & $\dddot{8}$ & छొ & $\stackrel{8}{\mathscr{C}}$ & 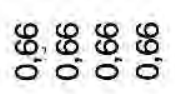 & 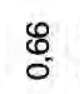 & 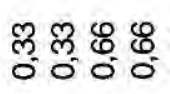 & 象 & $\stackrel{8}{0}$ \\
\hline 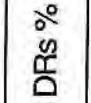 & 守 & 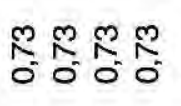 & $\frac{m}{0}$ & $\frac{m}{0}$ & $\frac{\mathscr{R}}{0}$ & $\frac{M}{0}$ & 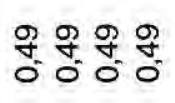 & 昌 & 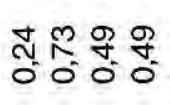 & 용 & 早 \\
\hline 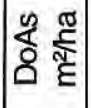 & $\begin{array}{l}\text { ठ্. } \\
\text { ర్ల }\end{array}$ & 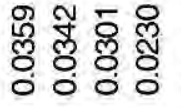 & 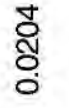 & $\begin{array}{l}8 \\
8 \\
0 \\
0\end{array}$ & $\begin{array}{l}\text { đ̊ } \\
\text { O } \\
0\end{array}$ & $\stackrel{\text { Y }}{\leftarrow}$ & 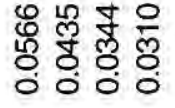 & 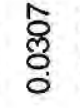 & 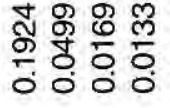 & 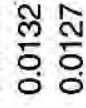 & Nิ \\
\hline $\begin{array}{l}\text { है } \\
\text { ठू } \\
\text { षै }\end{array}$ & $\frac{N}{0}$ & \begin{tabular}{llll}
0 & \multicolumn{1}{c}{} & 0 & 1 \\
0 & $\frac{1}{0}$ & $\frac{1}{0}$ & 0 \\
0 & 0 & 0 & 0
\end{tabular} & $\frac{}{0}$ & $\begin{array}{l}\text { 유 } \\
\text { ㅇ․ }\end{array}$ & $\frac{N}{\circ}$ & ¿̂̀ & 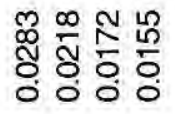 & $\frac{⿱ 亠 \pi}{0}$ & 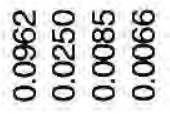 & 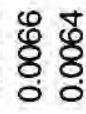 & $\begin{array}{l}\dddot{8} \\
8 \\
0\end{array}$ \\
\hline$\frac{0}{4}$ & $N$ & 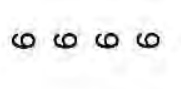 & $\omega$ & $\nabla$ & $\nabla$ & $\forall$ & $\forall ナ ナ ナ$ & $\forall$ & $\sim \sim \nabla+$ & $\forall \forall$ & $\nabla$ \\
\hline 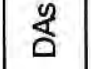 & $\nabla$ & 0060 & $\omega$ & $\omega$ & 0 & $\omega$ & オ オ & $\forall$ & $N 6 \nabla \forall$ & $\forall \nabla$ & $\checkmark$ \\
\hline ? & $N$ & $m m m$ & $m$ & $m$ & $m$ & $m$ & $\sim \sim N \sim$ & $N$ & $-M \sim N$ & $N N$ & $\sim$ \\
\hline$\frac{2}{5}$ & 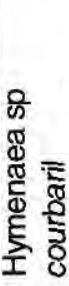 & 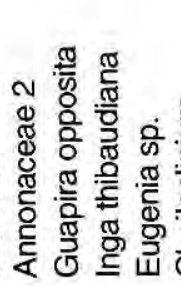 & 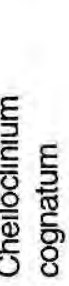 & 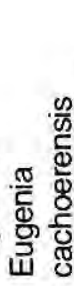 & 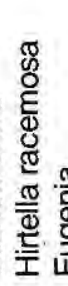 & 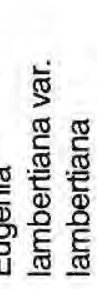 & 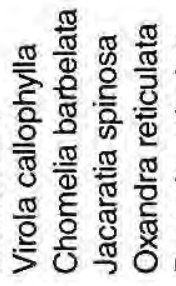 & 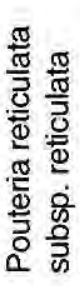 & 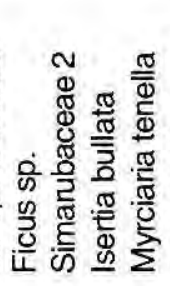 & 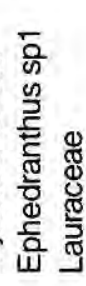 & 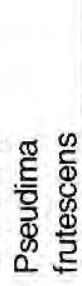 \\
\hline
\end{tabular}


cont. Tabela 1.

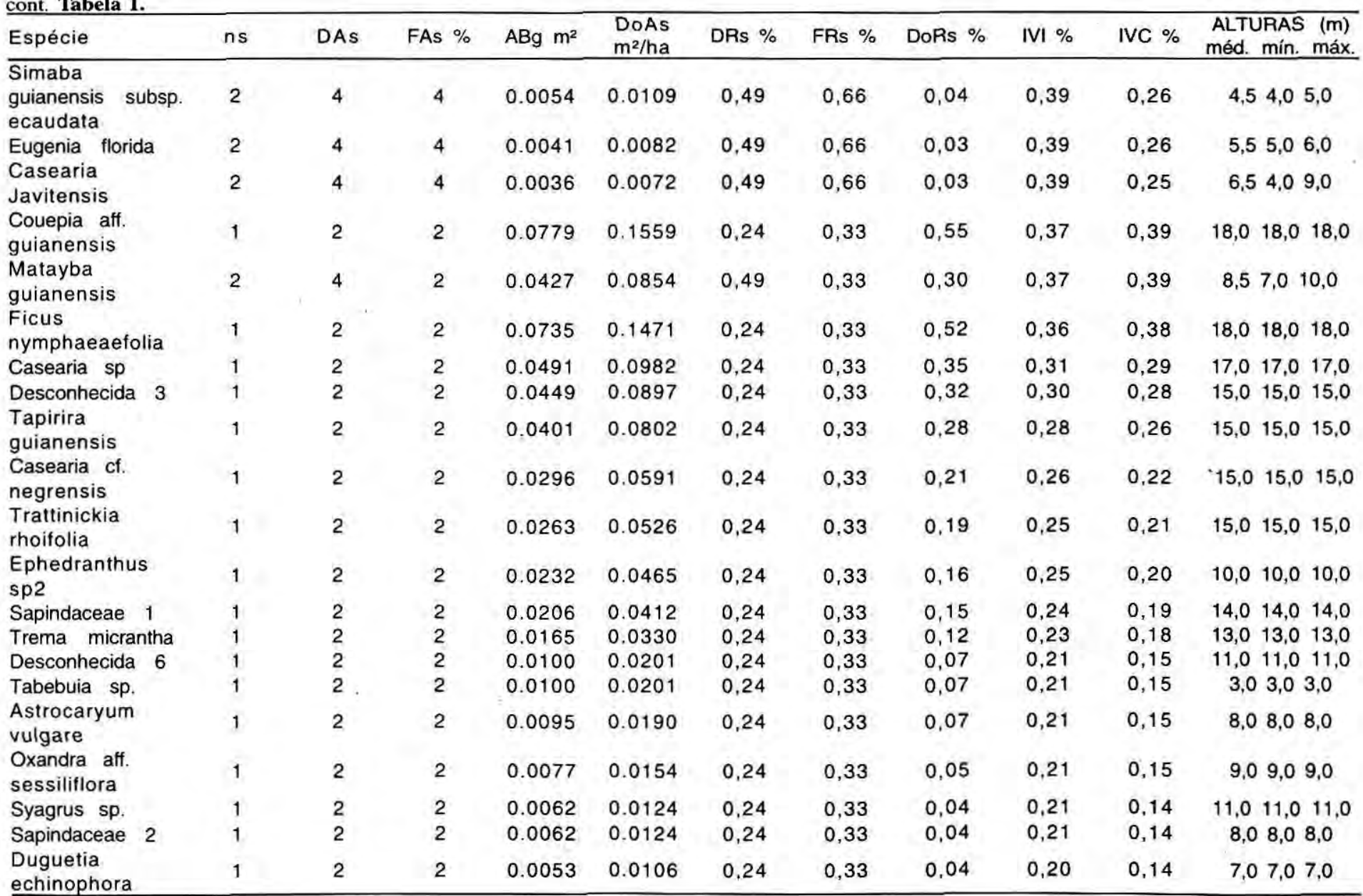




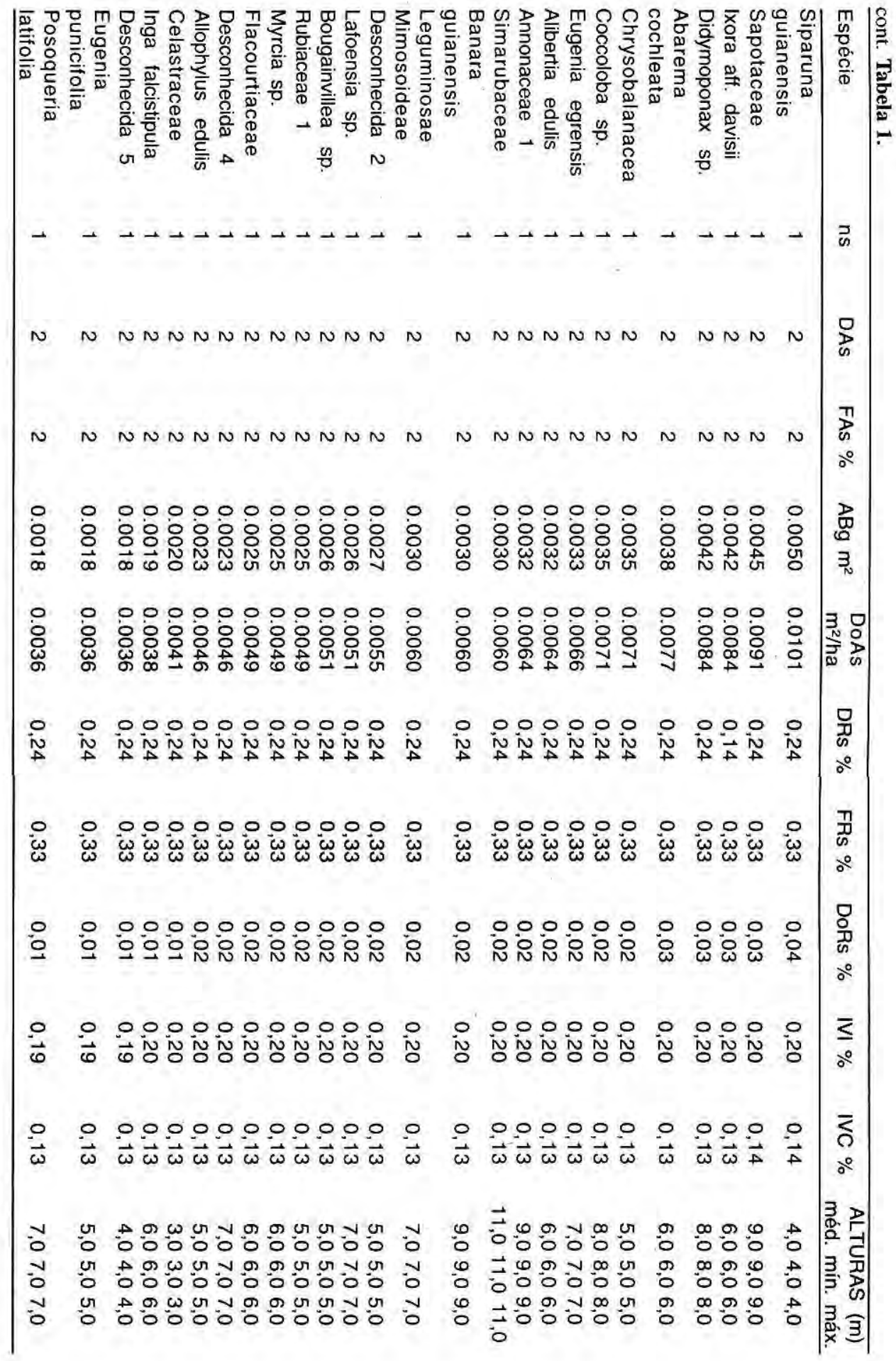


contribuição em todos os parâmetros em questão, devido principalmente aos valores apresentados por Copaifera langsdorffii, Dipteryx lacunifera, e $C$. officinalis. As Myrtaceae aparecem em segundo lugar tanto em freqüência quanto em densidade relativa, devido aos valores apresentados por Myrcia amazonica, $M$. cuprea, $M$. neesiana, uma espécie não identificada (Myrtaceae 1) e Campomanesia aromatica, não apresentando dominância expressiva.

A família Chrysobalanaceae, terceira em freqüência e densidade relativas, graças aos valores apresentados nesses parâmetros por Licania cf. incana e L. kunthiana, aparece em segundo lugar em dominância relativa, devido principalmente à grande contribuição de $L$. cf. incana, a mais expressiva de toda a fitocenose neste aspecto.

As Annonaceae aparecem nas quarta e quinta posições, respectivamente, em frequiencia e densidade relativas, devido principalmente à Annona montana. Meliaceae, quinta, quarta e terceira colocada em frequiência, densidade e dominância relativas, respectivamente, deve sua importância à Guarea guidonia em frequiência e densidade e à Carapa guianensis em dominância, sendo esta a segunda mais importante da fitocenose neste parâmetro, com apenas quatro indivíduos.

A familia Moraceae, que é apenas a décima terceira em frequiência relativa e a décima quinta em densidade relativa, se destaca em dominância relativa, sendo a quarta neste parâmetro, devido aos altos valores de área basal apresentados por Ficus citrifolia com apenas 2 indivíduos e Artocarpus integrifolia, com 4 árvores. Arecaceae, quinta colocada em dominância relativa, devido principalmente ao valor apresentado por Maximiliana maripa, assume a sétima posição em frequiência e a sexta em densidade relativas. Burseraceae deve sua sexta e sétima posições em freqüência e densidade relativas, respectivamente, a Protium heptaphyllum, com 15 indivíduos em 12 parcelas.

Com relação à frequiência e densidade relativas, a partir da quarta posição e até a décima oitava e décima sétima, respectivamente, nota-se uma inversão de posições, indicando que os parâmetros em questão estão mais ou menos distribuídos entre essas famílias. Vinte e duas famílias restantes contribuem com $18,03 \%$ da frequêencia relativa enquanto que 23 respondem por $13,37 \%$ da densidade relativa.

No tocante à dominância relativa, a importância é mais concentrada, uma vez que apenas 9 famílias são responsáveis por $83,36 \%$ do total, ficando as 31 famílias restantes com apenas $16,64 \%$.

\section{Índices de Valor de Importância (IVI) e de Cobertura (IVC)}

A Figura 2 mostra a distribuição dos índices de valor de importância (2A) e de cobertura (2B), por família, considerando-se aquelas que atingiram aproximadamente $82,0 \%$ do IVI e do IVC total.

Verifica-se que, até a quarta posição, não há inversão de posições, 

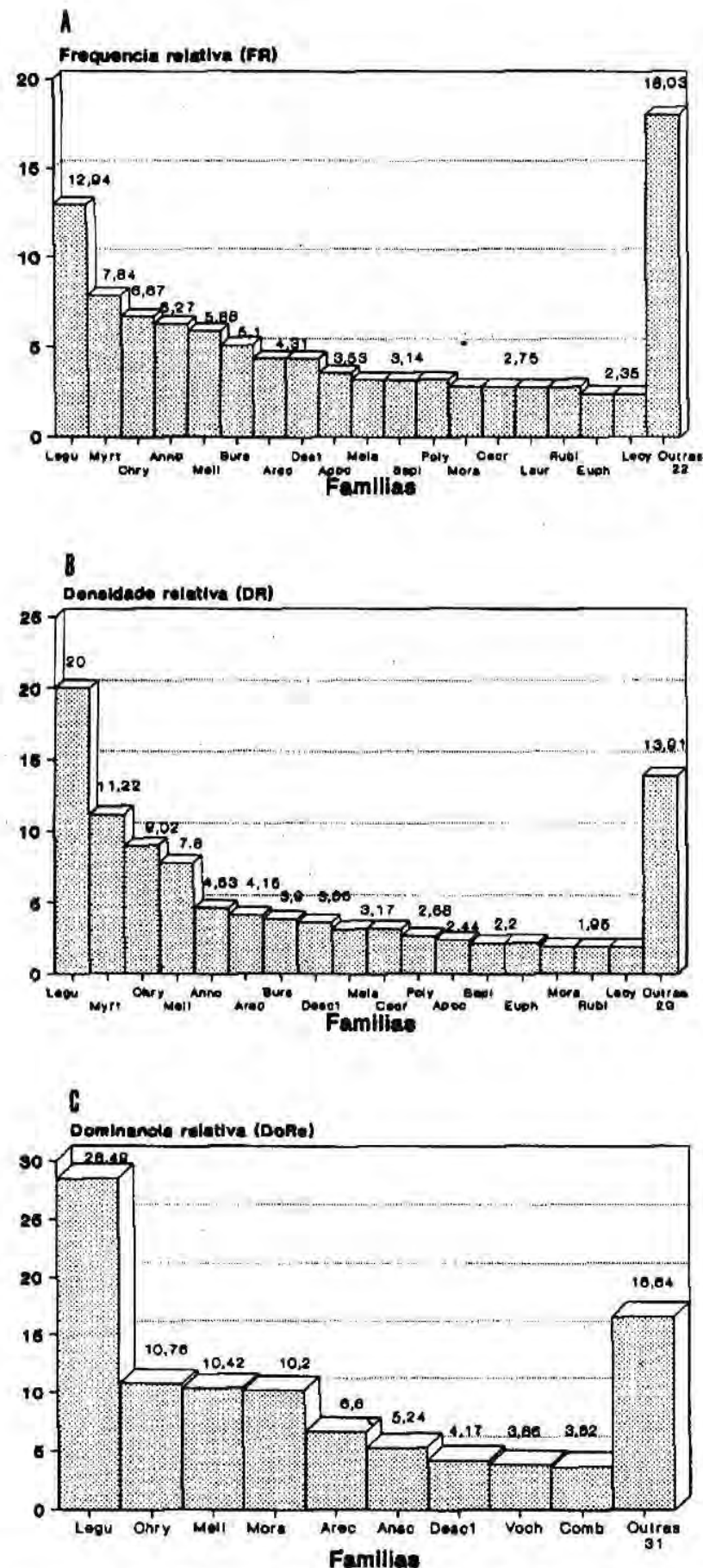

Figura 1. Distribuição por família de: A - frequência relativa; B - densidade relativa; C - dominância relativa. 
sendo as famílias Leguminosae, Chrysobalanaceae, Meliaceae e Myrtaceae, por ordem, as mais importantes, tanto em IVI quanto em IVC. As Leguminosae são de longe as mais importantes, principalmente devido à contribuição dada por Copaifera langsdorffii, Dipteryx lacunifera e $C$. officinalis .

Em seguida, e com valores mais baixos, aparecem as Chrysobalanaceae tendo em Licania cf. incana a principal contribuição; as Meliaceae, devido principalmente à Guarea guidonia e Carapa guianensis. As Myrtaceae devem sua importância a um grupo de espécies: Myrcia amazonica, $M$. cuprea, $M$. neesiana, uma espécie não identificada (Myrtaceae Indet.) e Campomanesia aromatica.

As famílias Arecaceate e Moraceae invertem as posições em IVI e IVC; a primeira é quinta em IVI e sexta em IVC, enquanto que a última ocupa a sexta posição em IVI e quinta em IVC. Esta inversão se deve principalmente à dominância de Maximiliana maripa, aliada a valores de densidade e freqüência relativamente altos para as Arecaceae, enquanto que os principais representantes das Moraceae, Ficus citrifolic e Antocarpus integrifolia, apresentam alta a média dominância, embora a freqüência e densidade sejam praticamente despreziveis.

Annonaceae ocupa a sétima posiçăo em IVI e oitava em IVC, invertendo as posições com Desconhecida 1 . Burseraceae é a nona em IVI e décima em IVC, cedendo a nona posição em IVC para Anacardiaceae, que é apenas a décima primeira em IVI. Outras 24 famílias somam 53,28 de IVI, equivalente a $17,77 \%$ do total, das quais 17 apresentam menos de 3,00 de IVI $(1,00 \%)$.

Considerando o IVC, 27 famílias restantes somam 37,68 (18,84\% do total). Dessas, 18 famílias apresentam menos de 2,00 de IVC $(1,00 \%)$.

Pela análise da Figura 3, que relaciona o número de espécies adicionais por parcela, acumulativamente, percebe-se que espécies são adicionadas gradativamente à comunidade à medida que se aumenta o número de parcelas, sem que haja uma tendência acentuada à estabilização.

\section{DISCUSSÃO}

A densidade total de 820 árvores/ha estimada para a área em estudo é alta, quando comparada com outros resultados observados nas florestas amazônicas (MUNIZ et al., 1994). Isto pode ser explicado pela escolha do DAP mínimo $(4,8 \mathrm{~cm})$, que acarreta a inclusão na amostra de indivíduos de dimensões menores, representando $45,36 \%$ do total. Quando se considera apenas os indivíduos com DAP a partir de $10 \mathrm{~cm}$, o número encontrado é de 448 árvores/ha, comparável, portanto, à média obtida em outros trabalhos. No entanto, este número é bem inferior ao encontrado por LISBOA (1989) em uma floresta secundária em Rondônia, usando o mesmo tamanho de área e o mesmo DAP mínimo, o que indica que a mata da Reserva Florestal do Sacavém é menos densa, quando comparada com as florestas amazônicas secundárias.

Outra característica desta mata é a densidade populacional de algumas espécies, como é o caso de Guarea 

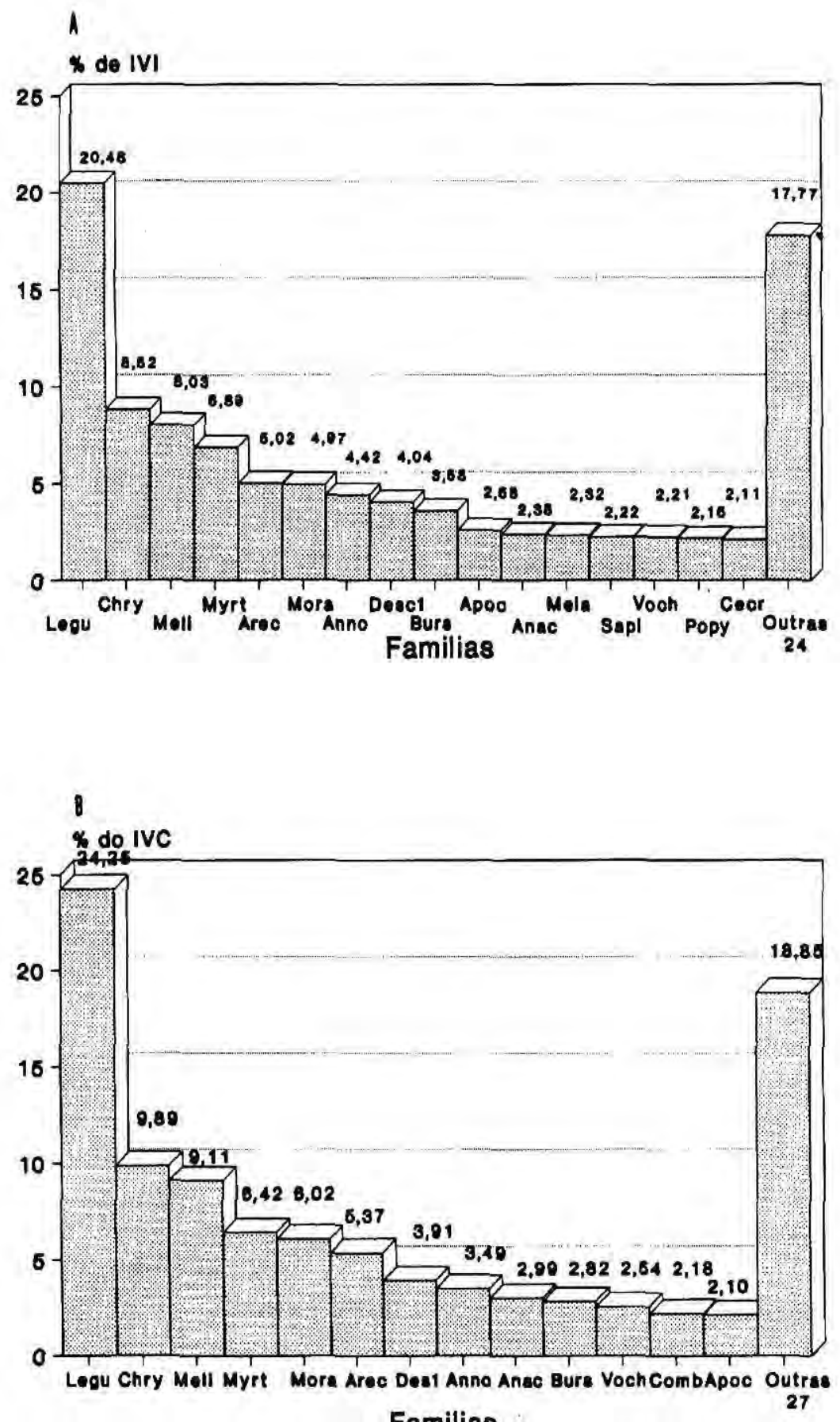

Figura 2. Distribuição por família de: $A$ - índice do valor de importância (IVI), perfazendo $82,23 \%$ do total; B - índice do valor de cobertura (IVC), perfazendo $81,15 \%$ do total. Reserva Florestal do Sacavém, São Luís - MA. 


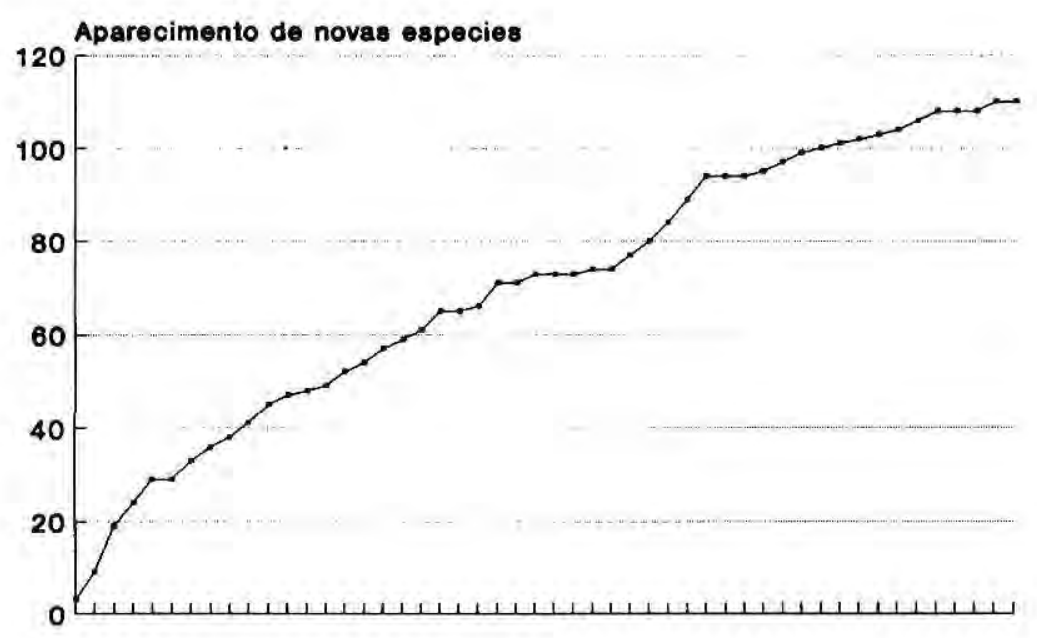

\section{Parcelas}

Figura 3. Curva acumulativa de espécies adicionais (espécie $x$ área), na Reserva Florestal do Sacavém, São Luís - MA.

guidonia, Licania cf. incana, Copaifera langsdorffii, Protium heptaphyllum, Desconhecida 1 , Dipteryx lacunifera, Copaifera officinalis, Cecropia palmata, Maximiliana maripa e Coccoloba pichuna. Estas 10 espécies contribuem com cerca de $40 \%$ da densidade total, enquanto que 65 espécies ( $60 \%$ das espécies consideradas) apresentam apenas 1 ou 2 indivíduos. Estes resultados são concordantes com as afirmativas de FEDOROV (1966) de que, em florestas tropicais úmidas, o número de espécies por unidade de área é muito alto, mas todas as espécies estão representadas por populações esparsas, sendo a densidade da maioria, via de regra, muito baixa. Segundo MORI et al. (1989), um número relativamente pequeno de espécies, contribui para a maioria do valor do índice de importância de uma dada floresta, e muitas espécies estão representadas por um ou poucos indivíduos por hectare. A resultados semelhantes já haviam chegado BLACK et al. (1950), segundo os quais a densidade populacional da metade ou mais das espécies arbóreas em florestas amazônicas é, provavelmente, menos que um indivíduo por hectare.

Quanto à densidade relativa, tomando-se as oito primeiras espécies, neste parâmetro (Tab. 1), observa-se que os valores não são muito discrepantes. Já quanto à freqüência relativa, considerando-se também as oito primeiras espécies, os valores são bem mais variáveis, embora, nenhuma espécie se destaque acentuadamente sobre outra. HEINSDIJK \& BASTOS (1963) afirmaram que, não obstante as variações de uma área para outra, a principal característica da floresta tropical úmida é o grande número de espécies e que, se uma ou algumas 
espécies apresentarem-se, acidentalmente, com freqüência capaz de levar a supor que se trata de formações puras, isto será apenas um aspecto local.

A freqüência está relacionada com a uniformidade ou regularidade com que os indivíduos de uma espécie estão distribuídos através da comunidade, isto é, tem a ver com homogeneidade e é expressa como a proporção de unidades de amostra que contêm uma determinada espécie. Devido ao relacionamento óbvio espécie-área, o tamanho da unidade amostral influencia a probabilidade de uma dada espécie estar presente na unidade. Portanto, a freqüência de uma espécie não é função apenas de sua densidade e padrão de ocorrência em uma comunidade, mas destas e do tamanho da área amostral juntos, sendo diretamente comparáveis apenas aquelas que tenham sido determinadas usando unidades amostrais de igual tamanho (CAIN \& CASTRO, 1959).

Em relação à dominância relativa, cujos valores são mais dependentes da biomassa, avaliada pela área basal dos indivíduos, as duas primeiras espécies são Licania cf. incana e Carapa guianensis, a primeira pela soma das áreas basais de todos os seus indivíduos, enquanto que a última, com bem menos indivíduos, apresenta um diâmetro individual bem maior.

Segundo RICHARDS (1952), cada localidade tem um conjunto característico de espécies dominantes, que não excede 20 em número. Freqüentemente a população consiste de uma a quatro espécies dominantes e de uma a seis espécies abundantes. As numerosas outras espécies estão representadas por únicos, ou muito poucos, indivíduos. As espécies dominantes e abundantes variam de local para local; uma espécie que é abundante em um local pode estar quase ausente em outro vizinho. Dessa maneira, a floresta é uma coleção de espécies de exigências ecológicas similares que ocorrem em combinação, flutuando na composição de um local para outro.

Comparando-se os valores de frequiência e densidade relativas por familia (Fig. 1A e 1B), observa-se que praticamente não há alteração entre as sete primeiras. Verifica-se que Leguminosae, Myrtaceae, Chrysobalanaceae, Meliaceae, Annonaceae, Arecaceae e Burseraceae ocupam posições relevante em relação a esses parâmetros. No que diz respeito à dominância relativa (Fig. $1 \mathrm{C}$ ), ressaltamse, além das Leguminosae, as Chrysobalanaceae, Meliaceae, Moraceae e Arecaceae, menos numerosas porém com mais biomassa, o que lhes confere maior representatividade neste parâmetro.

Para a Amazônia, nos levantamentos realizados, as maiores densidades são das famílias Arecaceae, Bignoniaceae, Burseraceae, Cochlospermaceae, Combretaceae, Euphorbiaceae, Lauraceae, Lecythidaceae, Leguminosae, Melastomataceae, Moraceae, Myrtaceae e Sapotaceae, enquanto que Arecaceae, Burseraceae, Caricaceae, Cochlospermaceae, Euphorbiaceae, Lecythidaceae, Leguminosae, Moraceae, Sapotaceae e Vochysiaceae se destacam em abundância.

O índice de valor de importância 
(IVI) das espécies tem valor muito relativo, uma vez que a dependência do tamanho, forma e número de parcelas é fundamental, não servindo, portanto, para comparar trabalhos com metodologias variadas (SALOMÃO \& LISBOA, 1988). De fato, em florestas equatoriais úmidas, a flora é tão rica, a densidade de uma determinada espécie tão baixa e a dominância tão baixa e variável, que estimativas da importância relativa são quase, se não realmente, impossíveis. O uso do IVI, entretanto, permite o arranjo de todas as espécies em uma série linear de acordo com os valores de seus índices (CAIN et al., 1956).

Comparando-se as posições das principais espécies quanto ao IVI e IVC, listadas na (Tab. 1), verifica-se que elas sofrem algumas alterações devido, principalmente, ao fato de que determinadas espécies têm maior densidade e dominância e baixa freqüência, ou vice-versa. Como se pode observar na mesma tabela, as 5 espécies mais importantes somam 68,12 pontos de IVI $(22,70 \%), 28$ espécies apresentaram valores de IVI maiores que $3,00(1 \%)$, perfazendo juntas $199,5(66,5 \%)$ dos 300 pontos e as demais 82 espécies somam os $100,5(33,5 \%)$ pontos restantes, o que torna claro a baixa significância relativa da maioria das espécies desta comunidade. Observa-se, também, que mesmo entre as espécies com maiores IVI, nenhuma se destaca acentuadamente sobre outra, o mesmo sendo válido a nível de família (Fig. 2A), com exceção das Leguminosae. Conseqüentemente, nenhuma espécie ou família pode ser considerada dominante, o que indica, ainda, que a importância entre as espécies e famílias, exceto Leguminosae, é igualmente distribuída. Este fato confirma as observações de RICHARDS (1952), que a maior parte da floresta primária consiste de associações mistas compostas de um número muito grande de espécies, nenhuma das quais é dominante sozinha, a grande maioria sendo representada por muito poucos indivíduos.

A floresta úmida é, dessa maneira, geralmente uma comunidade com um grande número de co-dominantes, embora algumas vêzes possa apresentar uma ou duas espécies dominantes. Os resultados aqui obtidos confirmam, também, o que foi dito por DUCKE \& BLACK (1954) que, depois das palmeiras, o elemento mais importante na fisionomia da flora hileana é constituído pelas Leguminosae. Segundo MORELLATO \& ROSA (1991) todos os tipos de vegetação possuem uma ou poucas espécies que dominam a maior parte dos espaços e, conseqüentemente, usufruem dos recursos disponíveis. Dessa forma, a riqueza em espécies está sendo determinada por muitas espécies representadas por 1 ou 2 indivíduos, com pequeno IVI.

Comparando-se com os resultados de outros levantamentos realizados na Amazônia (MUNIZ et al., 1994), as familias com maior IVI são Apocynaceae, Arecaceae, Burseraceae, Cochlospermaceae, Euphorbiaceae, Lauraceae, Lecythidaceae, Leguminosae, Meliaceae, Moraceae, Myrtaceae, Sapotaceae, Sterculiaceae, Tiliaceae e Vochysiaceae. SALOMÃO et al. (1988) afirmaram que 
não há diferenças marcantes entre os locais considerados e, sim, variaçōes no "ranking" das familias mais importantes, embora tenham salientado a necessidade de amostragens intensivas para posterior confirmação.

O significado da "dominância de familia" em florestas mistas, embora muito difundido, não é, absolutamente, conhecido. Geralmente acredita-se que a competição entre plantas ou animais é mais severa entre as espécies mais proximamente relacionadas. Sob este ponto de vista é surpreendente encontrar várias espécies, do mesmo gênero ou família, codominantes no mesmo habitat (RICHARDS, 1952). Assim, para a Reserva Florestal do Sacavém, não se observa dominância verdadeira, nem para espécies, nem para famílias, confirmando as numerosas observações feitas nas florestạs amazônicas.

A análise da curva acumulativa de espécies adicionais (Fig. 3) demonstra que a área amostrada deveria ter sido maior, embora, segundo BLACK et al. (1950), o número de espécies em uma amostra aleatória seja função de muitas variáveis, sendo as mais evidentes: o número total de espécies presentes no local onde, e quando, a amostra é feita; a abundância e a raridade das espécies; e o tamanho da área amostral. De fato, PIRES \& PRANCE (1985) afirmaram que na Floresta Amazônica, muitas espécies coexistem, sem que haja dominância verdadeira de uma sobre outra, e apenas um pequeno grupo responde por mais de $50 \%$ do número total de árvores, sendo a grande maioria representada apenas esporadicamente. Nessas condições quando se aumenta a área amostral o número de espécies aumenta gradualmente, de tal forma que a curva espécie-área nunca alcança a assintótica, porque sempre irão aparecer espécies encontradas pela primeira vez.

\section{PIRES \& KOURY} reafirmaram que a flora amazônica é tipicamente polimorfa e de grande porte, onde algumas espécies são raras, outras habitam determinadas áreas muito restritas, ainda outras podem ser dominantes em certos locais ou ainda, amplamente dispersas, sem que, na maioria dos casos, se conheça as leis que regulam estes fenômenos. Como caráter generalizado, entretanto, existem poucas espécies dominantes (representadas por muitos indivíduos) e muitas espécies raras (com poucos, e muito dispersos, indivíduos). PIRES et al. (1953) concluíram dizendo que, à medida que se aumenta a área amostral novas espécies são adicionadas, embora a taxa de adição se torne progressivamente mais lenta.

Pelas observações obtidas concluiuse que, para a mata da Reserva Florestal do Sacavém, em consequência da grande diversidade, a amostragem foi subdimensionada, ou seja, para um melhor conhecimento da flora local deveria-se aumentar a área amostral.

\section{Bibliografia citada}

BLACK, G.A.; DOBZHANSKY, T: PAVAN, C. 1950. Some attempts to estimate species diversity and population density of trees in Amazonian forest. Botanical Gazette, 111(4):413-425.

BRAUN-BLANQUET, J. 1979. Fitossociologia - bases para el estudio de las comunidades vegetales. Madrid, $\mathrm{H}$. Blume Ediciones. $440 \mathrm{p}$.

CAIN, S.A.; CASTRO, G.M. de O. 1959. Manual of vegetation analysis. New York, Harper 
and Brothers Publishers. 325p.

CAIM, S.A.; CASTRO, G.M. de O; PIRES, J.M.; SILVA, N.T. 1956. Application of some phytosociological tecniques to brazilian forest. American Journal of Botany, 43(10); 911941

CRONQUIST, A. 1981. An integrated system of classification of flowering plants. New York. Columbia University Press. 1262p.

DUCKE, A.; BLACK, G.A. 1954. Notas sobre a fitogeografía da Amazônia Brasileira. Boletim Técnico do Instituto Agronônico do Norte, 29:1-62.

ENGLER, A. 1964. Syllabus der pflanzenfamilien - angiospermen. Berlim, Gebruder Borntraeger. $666 \mathrm{p}$

FEDOROV, A.A. 1966. The structure of the tropical rain forest and speciation in the humid tropics. Joumal of Ecology, 54(1):1-11.

HEINSDIJK, D; BASTOS, A de M. 1963. Inventários florestais na Amazônia. Boletim do Serviço Florestal, 6:1-100.

LISBOA, P.L.B. 1989, Estudo f7orístico da vegetação arbórea de uma floresta secundária, em Rondônia. Boletim do Museu Paraense Emílio Goetdi, Botânica. 5(2):145-162.

MORELLATO, L.P.C.; ROSA, N.A. 1991. Caracterização de alguns tipos de vegetação na região amazônica, Serra dos Carajás, Pará, Brasil. Revista Brasileira de Botânica, 14:114.

MORI, S.A., et al. 1989. Composition and structure of an amazonian forest at Camaipi, Amapa, Brazil. Bolelin do Museu Paraense Emilio Goeldi, Botânica. 5(1):3-18.

MUELLER-DOMBOIS, D.; ELLENBERG, $\mathrm{H}$. 1974. Aims and methods of yegetation ecology: New York, Willey and Sons. 574p.
MUNIZ, F.H.; CESAR, O.; MONTEIRO, R. 1994. Aspectos florísticos quantitativos $\mathrm{e}$ comparativos da vegetação arbórea da Reserva Florestal do Sacavém, São Luís, Maranhão (Brasil). Revista Acta Amazonica. 24 (V2):

OREN, D.C. 1988. Uma reserva biológica para o Maranhão. Ciência Hoje, 44(8):36-45.

PIRES, J.M.; DOBZHANSKY, T:; BLACK, G.A. 1953. An estimate of the number of species of trees in an amazonian forest community. Botunical Gazette, 114:467-477.

PIRES, J,M.; KOURY, H.M. 1959. Estudo de um trecho de mata de várzea próximo a Belém. Boletim Técnico do Instituto Agronômico do Norte, 36:3-44.

PIRES, J,M; RANCE, G,T. 1985. The vegetation types of the Brazilian Amazon. In: PRANCE, G.T.; LOVEJOY, T.E. (ed.). Amazonia. Oxford, Pergamon Press. p.109145.

RICHARDS, P.W. 1952. The tropical rain forest: an ecological studty. New York, Cambridge University Press. $423 p$.

SALOMÃO, R. de P.; LISBOA, P.L.B. 1988. Análise ecológica da vegetação de uma floresta pluvial tropical de terra firme, Rondônia. Boletim do Museu Paraense Emítio Goeldi, Botânica, 4(2): 195-233.

SALOMÃO, R. de P; LISBOA, P.L.B; SILVA, M.F.F. da; ROSA. N. de A. 1988. Inventário ecológico em floresta pluvial tropical de terra firme, Serra Norte, Carajás, Pará. Boletim do Museu Paraense Emílio Goeldi, Botânica, 4(1):1-46. 